\title{
An Analysis of Perceptual Instability During Haptic Texture Rendering
}

\author{
Seungmoon Choi and Hong Z. Tan \\ Haptic Interface Research Laboratory \\ Purdue University \\ 1285 EE Building, West Lafayette, IN 47907-1285 \\ \{chois,hongtan\}@purdue.edu
}

\begin{abstract}
This paper investigates the problem of perceived instability during haptic texture rendering. We focus on the perceptual analysis of the stability of textured surfaces rendered with a force-reflecting device. The method of limits is used to assess the detection thresholds for perceived instability in terms of the stiffness of the virtual textured surfaces. We varied texture rendering method, exploration mode, and the amplitude and spatial wavelength of a sinusoidal surface texture model. Our results show that the maximum stiffness value for perceptually stable texture rendering is quite small $(<0.45 \mathrm{~N} / \mathrm{mm})$ for the range of textured surfaces tested. We also found that the stiffness thresholds depend on many factors such as rendering method, exploration mode, inter-subject difference, and surface model. Our current and future work focuses on the sources of perceived instability during haptic texture rendering. With these studies, we hope to develop strategies that can mitigate the problem of perceived instability during texture and other types of haptic rendering.
\end{abstract}

\section{Introduction}

This paper reports our initial findings from an ongoing research program on haptic texture perception and rendering. Our long-term research objectives are to have a better understanding of how to characterize surface textures in physical and perceptual terms, and to develop procedurebased rendering algorithms that can effectively span the human texture perception space.

Although everyone has some notion of what texture is, the concept of texture is not clearly defined. Katz considered texture as the fine structure of a surface (microstructure), and as independent of the shape (macrostructure) of an object or surface [12]. The systematic study of haptic texture perception began about thirty years ago [22]. One topic that has been controversial is whether informa- tion about surface texture is encoded spatially or temporally. Both types of information are available during direct (fingerpad) exploration, but only temporal cues (vibration) are available during indirect (probe-mediated) exploration. Katz argued that vibration was a necessary condition for texture (particularly roughness) perception [12]. Katz's position was based on the observation that one could easily perceive the roughness of a surface by stroking a pencil across it, and that performance was degraded when the pencil was wrapped in cloth (thereby damping the vibration transmitted through the pencil). Using the fingerpad exploration method, early studies by Lederman and her colleagues argued that vibration served only to prevent the cessation of activity in the mechanoreceptor population [29]. Their experiments found that selective vibrotactile adaptation (which resulted in a change of magnitude estimation of vibration signals) did not alter the perceived roughness of metal gratings [21], and speed of hand movements (which presumably affected the frequency of vibration) barely affected perceived roughness [16]. Consistent with these findings, a "spatial-intensive" model was proposed for roughness perception based on neurophysiological data [3, 4]. Recently, Lederman and her colleagues have begun to investigate texture perception through the use of intermediate objects such as probes and (compliant or stiff) finger coverings. With the probe-mediated exploration method, a substantial effect of speed was found, thereby supporting a theory based on temporal coding of texture [14, 20].

The consensus that has emerged from these studies is that humans use temporal cues (vibration) while exploring surface textures via a probe. While the same temporal cues are available during fingerpad exploration, humans prefer to use intensive (depth of microstructures) and/or spatial (size of microstructures) cues instead [10]. Performance with bare fingerpad was better for tasks requiring spatial judgments (haptic object recognition), but roughness perception was very similar whether the direct or the indirect method was used $[18,19]$. In addition, neurophysiological and psychophysical data suggest that temporal cues are responsible 
for perception of very fine surface details (with interelement spacing below $1 \mathrm{~mm})[9,11,15]$. For very smooth surfaces, the probe method produced greater perceived roughness than the fingerpad method [13]. Therefore, probe-mediated surface texture perception should yield results similar to the direct method, with better performance expected for verysmall-scale (less than $1 \mathrm{~mm}$ ) surface features.

It follows that devices that emulate probe-mediated texture exploration should yield successful rendering of textured surfaces. In addition to its utility in enhancing the richness of haptically rendered objects, haptic rendering of texture allows for precise control of stimulus parameters in psychophysical studies. In the past few years, haptic rendering of texture has received increased attention from the haptics research community. Minsky's Sandpaper system was perhaps the first successful attempt at generating synthetic textures with a two DOF force-reflecting joystick [25, 26]. Minsky used a tangential force-gradient algorithm for 2D texture rendering, where the displayed force is in the plane of the textured surface and proportional to the gradient of the surface-height profile. Several successful implementations of texture rendering methods using three (or more) DOF force-reflecting devices have been also reported (for examples, see $[5,8,23,27,28])$.

To those of us who have felt a textured surface rendered with a sinusoidal profile, it is immediately clear that the perception of texture is often tainted by undesirable buzzing and other kinds of noise (see, for example, [31, 32]). We use the term "perceived instability" to refer to these sensations in general. Many studies have investigated the stability of haptic interfaces using engineering analysis based on control theory (for examples, see [1, 2, 7, 24]). However, the majority of these studies focus on the "hard wall" problem in which one-dimensional haptic interaction is assumed using surfaces lacking details such as texture. To the best of our knowledge, dedicated research into the instability problem of haptic texture rendering has not been reported. To fill this gap, this paper concentrates on the analysis of perceptual instability for a virtual textured surface rendered with a force-feedback device. We have taken a human-centered approach in the sense that the decision criteria regarding the stability of virtual textures are based on human perception. This approach is chosen because some of the unrealistic sensations may occur while a haptic interface is stable from the point of view of control theory.

The specific objectives of the current study are:

- To quantify the parameter space that results in perceptually stable haptic texture rendering;

- To study the effect of human interaction patterns on the perceived stability of haptically rendered textures;

- To investigate the effect of rendering methods on perceived stability of a textured surface; and
- To gather qualitative descriptions of different kinds of perceived instability associated with texture rendering.

\section{Methods}

\subsection{Apparatus}

The hardware used in all experiments consists of a PHANToM (Model 1.0A, with a stylus as an interaction tool and encoder gimbals for orientation sensing), and a Pentium II PC (400MHz, 128MB RAM). This model of PHANToM has a maximum nominal stiffness of $3.5 \mathrm{~N} / \mathrm{mm}$.

\subsection{Stimuli}

The virtual textured surfaces used in our experiments are one-dimensional sinusoidal gratings superimposed on a flat surface. This underlying flat surface is always positioned so that it coincides with the $x y$ plane located at $z=0$ in the PHANToM coordinate frame (see Fig. 1). The sinusoidal gratings can be described by $z=A \sin \left(\frac{2 \pi}{L} x\right)+A$, where $A$ and $L$ denote the amplitude and (spatial) wavelength, respectively (see Fig. 2). Sinusoidal gratings are frequently used as the basic building blocks for textured surfaces because any surface profile can be modeled by a Fourier series (see, for example, [20, 30, 32]).

Two rendering methods are used in the current study. Both use a spring model to calculate the magnitude of rendered force as $K \cdot d(t)$, where $K$ is the stiffness of the textured surface, and $d(t)$ is the penetration depth of the stylus at time $t$ (see Fig. 2). The penetration depth is calculated as

$$
d(t)=\left\{\begin{array}{cl}
0 & \text { if } z(t)>0 \\
A \sin \left(\frac{2 \pi}{L} x(t)\right)+A-z(t) & \text { if } z(t) \leq 0
\end{array},\right.
$$

where $(x(t), y(t), z(t))$ is the position of the tip of the stylus.

The two methods differ in the way the force directions are rendered. The first method, introduced by Massie [23], renders a force $\mathbf{F}_{\text {mag }}(t)$ with a constant direction normal to the underlying flat wall of the textured surface. The second method, proposed by Ho, Basdogan and Srinivasan [8], renders a force $\mathbf{F}_{\mathrm{vec}}(t)$ with varying directions such that it remains normal to the local micro-geometry of the sinusoidal texture model. Mathematically,

$$
\begin{aligned}
\mathbf{F}_{\mathrm{mag}}(t) & =K d(t) \mathbf{n}_{W}, \\
\mathbf{F}_{\mathrm{vec}}(t) & =K d(t) \mathbf{n}_{T}(x(t), y(t), z(t)),
\end{aligned}
$$

where $\mathbf{n}_{W}$ is the normal vector of the underlying flat wall, and $\mathbf{n}_{T}(x, y, z)$ is the normal vector of the textured surface at $(x, y, z)$ (see Fig. 2 and Eqn. 1). Note that $\mathbf{F}_{\text {mag }}(t)$ and $\mathbf{F}_{\text {vec }}(t)$ always lie in the $z x$ plane, and remain perpendicular to the gravity vector in the $-y$ direction. This layout minimizes the effect of gravity on rendered forces. 


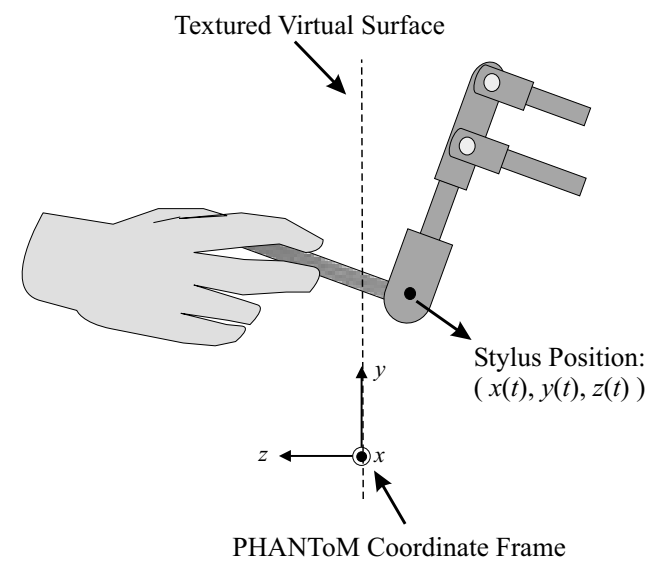

Figure 1. An illustration of the textured virtual surfaces used in our experiments. Note that $x$-axis comes out of the plane.

It follows from the above description that three parameters, $A, L$, and $K$, along with the texture rendering methods, uniquely define the stimuli used in this study.

Due to the fact that the workspace boundary of the PHANToM exhibits inferior dynamics performance, the virtual textured surface is restricted to a $15 \mathrm{~cm} \times 15 \mathrm{~cm}$ region located near the center of the PHANToM workspace.

\subsection{Subjects}

Three subjects participated in these experiments. One subject (SC, male) was also the experimenter, and was experienced with the PHANToM haptic interface. The other two subjects (SKG and SP, females) had not used any haptic interface prior to this study. The average age of the subjects was 26.3 years old. All subjects are right-handed and reported no known sensory or motor abnormalities with their upper extremities.

\subsection{Experimental conditions}

The independent variables employed in our experiments were rendering method, exploration mode, and amplitude and wavelength of sinusoidal surface profiles. As discussed earlier, two rendering methods, denoted by $\mathbf{F}_{\text {mag }}(t)$ and $\mathbf{F}_{\text {vec }}(t)$, were used. For exploration modes, we chose free exploration and stroking. With the free exploration mode, subjects were allowed to interact with the textured surfaces in whatever manner they desired. This mode was selected to be the most challenging interaction pattern for a haptic texture rendering system in terms of perceptual stability. With the stroking mode, subjects were instructed to move the stylus laterally across the textured surfaces (i.e., along the $x$

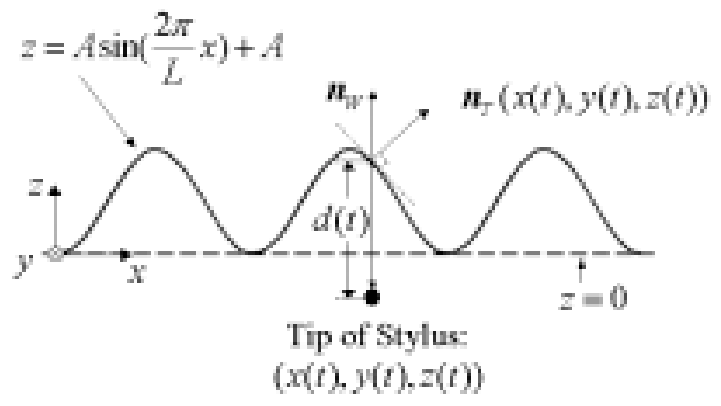

Figure 2. An illustration of the parameters used in texture rendering. Note that $y$-axis goes into the plane. See text for details.

axis as shown in Fig. 1). This mode was chosen to be representative of the typical and preferred exploration pattern for accurate texture perception [17]. Our four main experiments were thus defined by the combinations of the two texture rendering methods and the two exploration modes. For each of the four experiments, three values of $A(0.5$, 1.0 , and $2.0 \mathrm{~mm})$ and three values of $L(1.0,2.0$, and 4.0 $\mathrm{mm}$ ) were tested, resulting in a total of nine conditions per experiment (see Table 1).

The dependent variable measured in all 36 experimental conditions ( 2 rendering methods $\times 2$ exploration modes $\times$ $3 A$ values $\times 3 L$ values) was the maximum stiffness $K$ below which the rendered textured surface was perceived to be stable.

\subsection{Procedure}

The two inexperienced subjects (SKG and SP) went through initial training sessions to familiarize themselves with the PHANToM force-reflecting device, and to develop a criterion for perceived stability/instability of a virtual textured surface.

The method of limits was used in all experiments [6]. Given a pair of $A$ and $L$ values within each of the four experiments, a total of 100 series of trials (50 ascending series and 50 descending series) were conducted. Each ascending series started with a stiffness value of $K_{\min }=0.0$ $\mathrm{N} / \mathrm{mm}$ (i.e., no force) that was always perceived to be stable. The subject would respond "stable." The $K$ value was then increased by $\Delta K=0.02 \mathrm{~N} / \mathrm{mm}$. The subject would feel the virtual textured surface again and respond "stable" or "unstable." As long as the subject reported "stable," the $K$ value was increased by the same $\Delta K$ value for each subsequent trial. An ascending series was terminated when the subject reversed the response from "stable" to "unstable." The value of $K+\Delta K / 2$ was then recorded as the estimated 
Table 1. Experimental conditions

\begin{tabular}{c|c|c|c|c} 
Experiment & Texture Rendering Method & Exploration Mode & $A(\mathrm{~mm})$ & $L(\mathrm{~mm})$ \\
\hline \hline I & $\mathbf{F}_{\mathrm{mag}}(t)$ & Free Exploration & $0.5,1.0,2.0$ & $1.0,2.0,4.0$ \\
\hline II & $\mathbf{F}_{\mathrm{mag}}(t)$ & Stroking & $0.5,1.0,2.0$ & $1.0,2.0,4.0$ \\
\hline III & $\mathbf{F}_{\mathrm{vec}}(t)$ & Free Exploration & $0.5,1.0,2.0$ & $1.0,2.0,4.0$ \\
\hline IV & $\mathbf{F}_{\mathrm{vec}}(t)$ & Stroking & $0.5,1.0,2.0$ & $1.0,2.0,4.0$ \\
\hline
\end{tabular}

threshold for this ascending series, where $K$ is the stiffness of the last trial with a "stable" response.

Each descending series started with a stiffness value of $K_{\max }=0.6 \mathrm{~N} / \mathrm{mm}$. This value was selected based on the preliminary finding that no textured surface felt stable at this $K$ value. The same step size of $\Delta K=0.02 \mathrm{~N} / \mathrm{mm}$ was used to decrease $K$ values in each subsequent trial. A descending series was terminated when the subject reversed the response from "unstable" to "stable." The value of $K-\Delta K / 2$ was then recorded as the estimated threshold for this descending series, where $K$ is the stiffness of the last trial with an "unstable" response. With these chosen values of $K_{\min }$, $K_{\max }$ and $\Delta K$, each ascending/descending series could last up to 31 trials.

The experiments proceeded as follows. Each subject performed all nine conditions ( $3 A$ values $\times 3 L$ values) in Exp. I first, followed by those in Exps. II, III, and IV. For each pair of $A$ and $L$ values, the order of the 50 ascending and 50 descending series was randomized. The order of the nine conditions within each experiment was also randomized for each subject.

During all experiments, subjects wore headphones with white noise to block the auditory cues emanating from the PHANToM. No visual rendering of the textured surface was provided. Instead, the computer monitor only displayed text information on the series number.

The following instructions were given to the subjects during all experiments. They were asked to hold the stylus lightly, and to hold it like a pencil. For the free exploration mode (Exps. I and III), the subjects were asked to detect any "unrealistic vibration" as an indication of instability. For the stroking mode (Exps. II and IV), the subjects were instructed to concentrate on the detection of "unrealistic vibration" that occurred while they moved the stylus back and forth along the $x$ direction across the textured surface. They were asked to ignore other sensations such as perceived instability near the plane $z=0$ (i.e., when the stylus entered and left the textured surface). Subjects were asked to maintain a constant stroking velocity to the best of their ability.

Subjects were allowed to take a break whenever they desired. A ten-minute break was enforced after a subject had completed the 100 ascending/descending series for each experimental condition. This was found to be necessary in or- der to prevent the carryover effect (i.e., surfaces presented after a series of particularly unstable conditions might have been judged as more stable). Typically, each subject finished two or three experimental conditions per day.

\subsection{Stability of nontextured flat wall}

After the completion of the main experiments, one subject (SC) was tested with a nontextured flat wall with the same method of limits procedure as described above. The result of this post-testing served to provide a reference value for the stiffness $K$. It was expected that the threshold found in this post-testing would be larger than the ones obtained with textured surfaces.

\section{Results}

In this section, we first describe how data for each experimental condition were combined to obtain a threshold value for $K$. We then present individual and averaged threshold data for all subjects and all experiments, with results of statistical analysis.

As discussed earlier in the method section, 50 ascending and 50 descending series were conducted for each experimental condition (i.e., each pair of $A$ and $L$ values within a main experiment). Fig. 3 shows typical results for one experimental condition (Subject SC, $\mathbf{F}_{\text {mag }}(t)$, stroking, $A=2.0$ $\mathrm{mm}$, and $L=2.0 \mathrm{~mm}$ ). The top panel shows the histogram for all 50 ascending series, the middle panel for all 50 descending series, and the bottom panel for combined series. The mean $K$ value from the 50 ascending series $(0.26$ $\mathrm{N} / \mathrm{mm})$ is greater than that from the descending series $(0.19$ $\mathrm{N} / \mathrm{mm}$ ). This is typical and reflects what is termed the "errors of habituation" [6]. It is a common practice to compute the mean from the combined data $(0.23 \mathrm{~N} / \mathrm{mm})$ and regard it as an estimate of the stiffness threshold, $K_{T}$. We also calculated the standard deviation from the combined data. Note that this results in an over-estimated standard deviation (especially in the cases where the means associated with the ascending and descending series are very different).

The stiffness thresholds for one subject (SC) for Exp. IV are shown in Fig. 4. The values of $K_{T}$ for the nine experimental conditions are plotted in a three-parameter Cartesian 

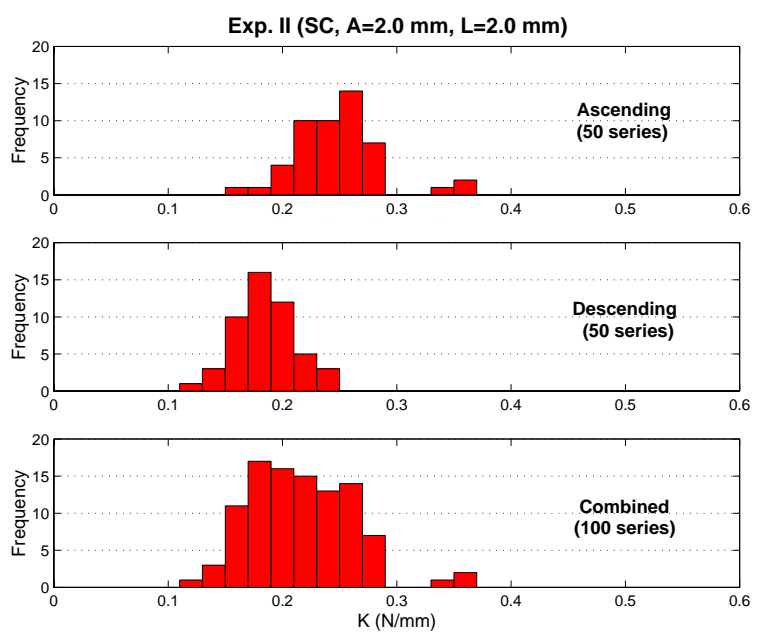
Figure 3. Typical histograms for one experi-
mental condition.

space, $\left(A, L, K_{T}\right)$. A fitted surface computed by linear regression analysis (with $\log _{2} A$ and $\log _{2} L$ as the variables, see the last two paragraphs of this section for details) is also shown to represent the region within which virtual textured surfaces are guaranteed to be perceptually stable. To help the reader visualize the spatial relationship between threshold data points (open squares) and the fitted surface (mesh), straight lines are drawn between the centers of data points and the corresponding points on the mesh with the same $A$ and $L$ values. The standard errors are not indicated in the figure because they are very small (the average standard error $=0.00482 \mathrm{~N} / \mathrm{mm}$ ).

Five-way ANOVA analysis (subject, texture rendering method, exploration mode, $A$, and $L$ ) shows that all five factors are statistically significant for the values of $K_{T}$. In particular, there are significant differences among the three subjects tested $(F(2,10791)=484.57, p<0.0001)$. Despite the significant inter-subject differences on the levels of $K_{T}$, however, the general trends exhibited by the data are common among the subjects. We thus make the following observations with the data pooled from all subjects.

Data pooled from all the subjects are shown in Fig. 5 along with the best-fitting surfaces. Several trends are immediately apparent from Fig. 5. First of all, the values of $K_{T}$ ranges from $0.0138 \mathrm{~N} / \mathrm{mm}$ to $0.4527 \mathrm{~N} / \mathrm{mm}$ for all the conditions tested. These values are quite small and the resulting textured surfaces feel very soft. They are also much smaller than the stiffness threshold measured with a nontextured wall $(1.005 \pm 0.157 \mathrm{~N} / \mathrm{mm}$ for subject SC). Second, the thresholds associated with the stroking mode (Exps. II and IV) are larger than those associated with the free-exploration mode (Exps. I and III) by the average of $0.137 \mathrm{~N} / \mathrm{mm}(F(1,10764)=5980.13, p<0.0001)$. Third,
Exp. IV (SC)

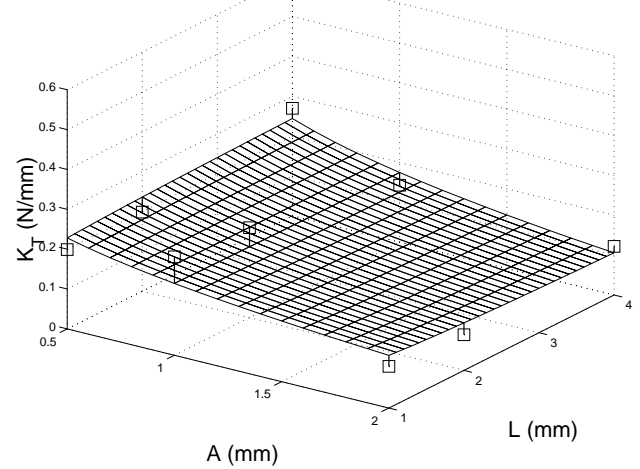

Figure 4. Stiffness thresholds (open squares) for Exp. IV (subject SC). Standard errors are not shown because they are too small to be visible. See text for details.

\section{Table 2. Coefficients of fitted regression equation.}

\begin{tabular}{c|c|c|c|c} 
Experiment & $\beta_{0}$ & $\beta_{A}$ & $\beta_{L}$ & $\beta_{A L}$ \\
\hline \hline I & 0.10724 & -0.04756 & 0.0 & 0.00709 \\
\hline II & 0.28116 & -0.15598 & 0.0 & 0.00709 \\
\hline III & 0.01226 & 0.00711 & 0.03309 & -0.03338 \\
\hline IV & 0.13591 & -0.03410 & 0.00966 & -0.03338 \\
\hline
\end{tabular}

the thresholds for surfaces rendered with the $\mathbf{F}_{\text {mag }}(t)$ method (Exps. I and II) are statistically greater than those with the $\mathbf{F}_{\text {vec }}(t)$ method (Exps. III and IV) by the average of 0.099 $\mathrm{N} / \mathrm{mm}(F(1,10764)=3103.44, p<0.0001)$.

A functional relationship between $(A, L)$ and $K_{T}$ are estimated using the following form of a fitted equation.

$$
\hat{K}_{T}=\beta_{0}+\beta_{A} \log _{2} A+\beta_{L} \log _{2} L+\beta_{A L} \log _{2} A \cdot \log _{2} L .
$$

The estimated coefficients are listed in Table 2 for all the experiments. They are computed by linear regression analysis for $K_{T}$ with two $\log$-scaled continuous variables $\left(\log _{2} A\right.$ and $\log _{2} L$ ) and two categorical variables (texture rendering method and exploration mode) as well as their interaction terms $\left(R^{2}=0.5908 . R^{2}\right.$ is relatively small because we pooled the data of all subjects that are significantly different). Note that the statistically insignificant coefficients are set to zero in this table.

The effects of the amplitude $(A)$ and wavelength $(L)$ of the sinusoidal gratings on the stiffness threshold $K_{T}$ are more complex. In Exps. I and II, it is evident that $K_{T}$ decreases as $A$ increases. The wavelength $(L)$ has an effect on $K_{T}$ only through the interaction term $\log _{2} A \cdot \log _{2} L$, but its effect is very small compared to that of $A\left(\beta_{L}=0.0\right.$, 
Exp. I

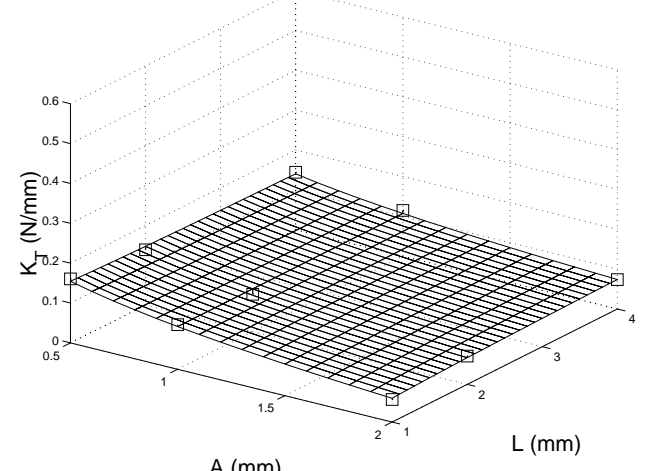

Exp. III



Exp. II

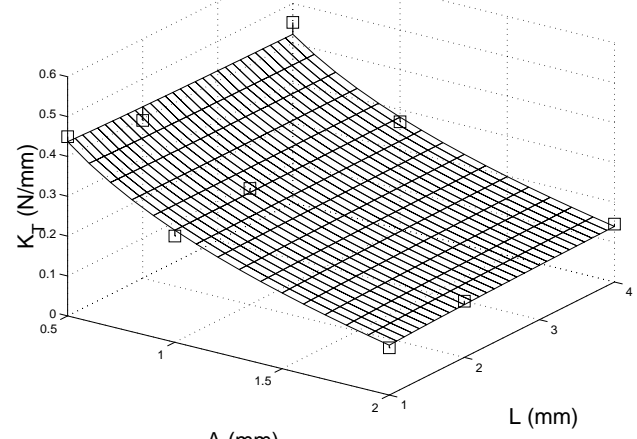

Exp. IV

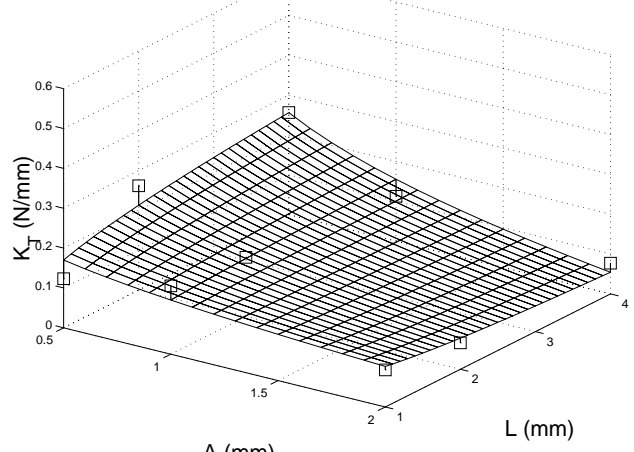

$A(\mathrm{~mm})$

Figure 5. Regression surfaces representing the boundary of $\mathrm{K}$ for perceptually stable texture rendering. Error bars are not shown as they are too small to be visible in these plots. Each datum point is averaged over all three subjects. A solid line is drawn between the center of the datum point and the corresponding point on the regression surface with the same $A$ and $L$ values to help visualize the position of the datum point.

$\left.\left|\beta_{A L}\right| \ll\left|\beta_{A}\right|\right)$. On the other hand, both $A$ and $L$ are significant factors for the values of $K_{T}$ in Exps. III and IV, and their interaction is more apparent $\left(\left|\beta_{A L}\right|\right.$ of Exp. III and IV $\gg\left|\beta_{A L}\right|$ of Exp. I and II). In Exp. III, increasing $A$ or $L$ tends to result in lower or higher $K_{T}$, respectively, unless $K_{T}$ is very small. In Exp. IV, the $K_{T}$ values and the regression surface show more complex dependence on $A$ and $L$.

\section{Discussion}

We set out to define a parameter space within which haptic rendering of textured virtual surfaces are guaranteed to be stable not only mechanically but perceptually. We have found, however, that such a space is quite limited. For nine sinusoidal surface profiles with an amplitude range of 0.5 to $2.0 \mathrm{~mm}$ and a spatial wavelength of 1.0 to $4.0 \mathrm{~mm}$, the maximum stiffness values that result in perceptually stable tex- tured surfaces are limited to $0.45 \mathrm{~N} / \mathrm{mm}$ or less. This stiffness range corresponds to surfaces that are soft and spongy to the touch. We also found that the region of parameter space for perceptually stable texture rendering depends on the rendering method and interaction pattern. A simple spring model with a fixed force direction results in more perceptually stable textured surfaces than one with varying force directions based on the local surface normals. This is consistent with the observations made in [8]. Virtual surfaces explored with lateral stroking are perceived to be more stable than those with a free interaction style. The amplitude of the sinusoidal texture model is the dominant parameter for the stiffness thresholds when the textures are rendered with fixed force directions. Both amplitude and wavelength are significant for the virtual textures with varying force directions based on local texture normals.

Subject debriefing has revealed several types of perceived instability during haptic texture rendering. The first 
and the most prevalent is entry-point instability. As the stylus approaches a point on the $z=0$ plane (see Fig. 1), one experiences "buzzing" of the stylus. The second type is associated with poking. It is most evident when the stylus is pushed deep into the virtual surface. The third type is related to the fact that it is difficult to hold a stylus still in a 3-D space. This results in changes in the measured position/orientation of the stylus, and consequently the rendered force. One therefore experiences variations in force while the stylus is perceived to be held still. The last type of perceived instability is due to the non-homogeneous dynamics of the PHANToM across its workspace. We have observed that in addition to the expected poor performance near its workspace boundary, the PHANToM seems to have a structural resonance near the center of its workspace. This is consistent with anecdotal notes from other researchers (e.g., personal communication with Vincent Hayward, 2000).

There are several other factors that we had no control over, but could have contributed to the perceived stability/instability of virtual textured surfaces. They include the grip of the stylus (both position and force), the angle at which the stylus is held against a virtual surface, and the speed at which the stylus is stroked across a textured surface.

In an effort to understand the sources of perceived instability, we have instrumented the last link (i.e., the link closest to the stylus) of the PHANToM with a triaxial force/torque sensor. We have also attached a triaxial accelerometer to the stylus. Position, force and acceleration measurements are taken under various conditions (rendering method, exploration mode, $A$ and $L$ values, and perceived stability/instability). Preliminary data analysis reveals a strong spectral component in the frequency range of $200-250 \mathrm{~Hz}$ in the position, force and acceleration data whenever textured surface are perceived to be unstable. This seems to be consistent with the authors' observation that the buzzing or noise is of high (vibrational) frequency. Our current work focuses on the further analysis of these measurement data. In particular, we will quantify the intensity of various spectral components in terms of sensation level (i.e., decibels above the corresponding absolute detection threshold of the vibration at the same frequency). We also plan to measure the dynamics of the PHANToM at several workspace locations in the near future, with the goal to locate the source of this high-frequency spectral component that could have contributed to the perceived instability of virtual textured surfaces.

\section{Acknowledgement}

This work was supported in part by a National Science Foundation (NSF) Faculty Early Career Development (CAREER) Award under Grant 9984991-IIS, and in part by an
NSF award under Grant 0098443-IIS.

\section{References}

[1] R. J. Adams and B. Hannaford. Stable haptic interaction with virtual environments. IEEE Transactions on Robotics and Automation, 15(3):465-474, June 1999.

[2] J. E. Colgate, M. C. Stanley, and J. M. Brown. Issues in the haptic display of tool use. In Proceedings of IEEE/RSJ International Conference on Intelligent Robots and Systems, pp. 140-145, 1995.

[3] C. E. Connor, S. S. Hsiao, J. R. Phillips, and K. O. Johnson. Tactile roughness: Neural codes that account for psychophysical magnitude estimates. Journal of Neuroscience, 10:3823-3836, 1990.

[4] C. E. Connor and K. O. Johnson. Neural coding of tactile texture: Comparison of spatial and temporal mechanisms for roughness perception. Journal of Neuroscience, 12:34143426, 1992.

[5] J. P. Fritz and K. E. Barner. Stochastic models for haptic texture. In Proceedings of SPIE's International Symposium on Intelligent Systems and Advanced Manufacturing - Telemanipulator and Telepresence Technologies III. Boston, MA, 1996.

[6] G. A. Gescheider. Psychophysics: Method, Theory, and Application. Lawrence Erlbaum Associates, Inc., Publishers, second edition, 1985.

[7] R. B. Gillespie and M. R. Cutkosky. Stable user-specific haptic rendering of the virtual wall. In Proceedings of the International Mechanical Engineering Congress and Exhibition, vol. 58, pp. 397-406, Atlanta, GA, 1996. ASME.

[8] C. Ho, C. Basdogan, and M. A. Srinivasan. Efficient pointbased rendering techniques for haptic display of virtual objects. Presence, 8(5):477-491, 1999.

[9] M. Hollins and S. R. Risner. Evidence for the duplex theory of tactile texture perception. Perception $\mathcal{F}$ Psychophysics, 62:695-705, 2000.

[10] K. O. Johnson and S. S. Hsiao. Neural mechanisms of tactual form and texture perception. Annual Review of Neuroscience, 15:227-250, 1992.

[11] K. O. Johnson and S. S. Hsiao. Evaluation of the relative roles of slowly and rapidly adapting afferent fibers in roughness perception. Canadian Journal of Physiology $\mathcal{E}$ Pharmacology, 72:488-497, 1994.

[12] D. Katz. The World of Touch. Lawrence Erlbaum Associates, Hillsdale, NJ, 1925/1989.

[13] R. L. Klatzky and S. J. Lederman. Tactile roughness perception with a rigid link interposed between skin and surface. Perception $\mathcal{E}$ Psychophysics, 61:591-607, 1999.

[14] R. L. Klatzky, S. J. Lederman, C. Hamilton, and G. Ramsay. Perceiving roughness via a rigid probe: Effects of exploration speed. In Proceedings of the ASME Dynamic Systems and Control Division, vol. 67, pp. 27-33. ASME, 1999.

[15] R. H. LaMotte and M. A. Srinivasan. Surface microgeometry: Tactile perception and neural encoding. In O. Franzen and J. Westman, editors, Information Processing in the Somatosensory Systems, Wenner-Gren International Symposium Series, pp. 49-58. MacMillan Press, 1991. 
[16] S. J. Lederman. Tactual roughness perception: Spatial and temporal determinants. Canadian Journal of Psychology, 37:498-511, 1983.

[17] S. J. Lederman and R. L. Klatzky. Hand movement: A window into haptic object recognition. Cognitive Psychology, 19:342-368, 1987.

[18] S. J. Lederman and R. L. Klatzky. Feeling through a probe. In Proceedings of the ASME Dynamic Systems and Control Division, vol. 64, pp. 127-131. ASME, 1998.

[19] S. J. Lederman and R. L. Klatzky. Sensing and displaying spatially distributed fingertip forces in haptic interfaces for teleoperator and virtual environment systems. Presence, 8:86-103, 1999.

[20] S. J. Lederman, R. L. Klatzky, C. L. Hamilton, and G. I. Ramsay. Perceiving roughness via a rigid probe: Psychophysical effects of exploration speed and mode of touch. Haptics-e (http://www.haptics-e.org), 1(1), 1999.

[21] S. J. Lederman, J. M. Loomis, and D. A. Williams. The role of vibration in the tactual perception of roughness. Perception $\mathcal{E}$ Psychophysics, 32:109-116, 1982.

[22] S. J. Lederman and M. M. Taylor. Fingertip force, surface geometry, and the perception of roughness by active touch. Perception $\mathcal{E}$ Psychophysics, 12:401-408, 1972.

[23] T. H. Massie. Initial haptic explorations with the phantom: Virtual touch through point interaction. Master's thesis, MIT, Feb. 1996.

[24] B. E. Miller, E. Colgate, and R. A. Freeman. Guaranteed stability of haptic systems with nonlinear virtual environments. IEEE Transactions on Robotics and Automation, 16(6):712719, 2000.
[25] M. Minsky and S. J. Lederman. Simulated haptic textures: Roughness. In Proceedings of the ASME Dynamic Systems and Control Division, vol. 58, pp. 421-426. ASME, 1996.

[26] M. D. R. Minsky. Computational Haptics: The Sandpaper System for Synthesizing Texture for a Force-Feedback Display. PhD thesis, MIT, June 1995.

[27] D. Ruspini, K. Kolarov, and O. Khatib. The haptic display of complex graphical environments. In Computer Graphics Proceedings, Annual Conference Series, pp. 345-352. ACM SIGGRAPH 97, 1997.

[28] J. Siira and D. K. Pai. Haptic texturing - a stochastic approach. In Proceedings of the IEEE International Conference on Robotics and Automation, pp. 557-562, 1996.

[29] M. M. Taylor and S. J. Lederman. Tactile roughness of grooved surfaces: A model and the effect of friction. Perception $\mathcal{F}$ Psychophysics, 17:23-36, 1975.

[30] S. A. Wall and W. S. Harwin. Modeling of surface identifying characteristics using Fourier series. In Proceedings of the ASME dynamic systems and control division, vol. 67, pp. 65-71, 1999.

[31] S. A. Wall and W. S. Harwin. Effects of physical bandwidth on perception of virtual gratings. In Proceedings of the ASME Dynamic Systems and Control Division, vol. 69, pp. 1033-1039, 2000.

[32] J. M. Weisenberger, M. J. Krier, and M. A. Rinker. Judging the orientation of sinusoidal and square-wave virtual gratings presented via 2-DOF and 3-DOF haptic interfaces. Haptics-e (http://www.haptics-e.org), 1(4), 2000. 